M. NERACHER

W. HUBSCHMID

\title{
Heterodyne-detected electrostrictive laser-induced gratings for gas-flow diagnostics
}

\author{
Paul Scherrer Institut, 5232 Villigen PSI, Switzerland
}

\begin{abstract}
Received: 19 March 2004/Revised version: 23 July 2004
Published online: 15 September 2004 • (C) Springer-Verlag 2004

ABSTRACT The signal of heterodyne-detected electrostrictive laser-induced gratings (LIGs) originates from the interference of a reference laser beam with the laser light diffracted at the counterpropagating sound-wave packets, which are generated in the overlap volume of crossed laser beams. The frequencies of the sound waves, which contain the information on the sound velocity and on the motion of the medium, can approximately be extracted from the frequencies at the maxima of the two peaks, which dominate the power spectrum of the heterodyne LIG signal intensity. In free-air jets behind a slot nozzle, flow velocities up to $60 \mathrm{~m} \mathrm{~s}^{-1}$ were determined by quick fits from the power spectrum and by fitting the time-dependent signal intensity data to model functions. Results agree well, the standard deviations being about one-half in the latter case $\left(1.6-2.6 \mathrm{~m} \mathrm{~s}^{-1}\right.$, for positions close to the nozzle). Problems arising from the sampling and from the finite observation time of the signal intensity are discussed. Furthermore, the results of the LIG measurements were compared with data provided by laser Doppler anemometry. As an application of the instantaneous and unseeded LIG measurement technique, temperatures in heated air jets were determined simultaneously with the flow velocity by quick fits from the power spectrum.
\end{abstract}

PACS 42.62.-b; 47.62.+q; 43.58.+z

\section{1}

\section{Introduction}

In many applications where flow parameters are measured, it is not possible to seed the flow with particles. Therefore, the applicability of the particle-tracer techniques like laser Doppler anemometry (LDA) [1] or particle-image velocimetry (PIV) [2] is limited. A strong interest to avoid seeding therefore exists. Several studies [3-14] in recent years have demonstrated the functionality of seedless diagnostics approaches in various flow regimes, employing electrostrictive and thermal laser-induced gratings (LIGs). The combination of LIGs with heterodyne detection [3] allows the simultaneous determination of flow velocity and temperature. Modelling of the processes of grating formation and grating interrogation is described in detail by Cummings et al. [3]

Fax:+41-56-310-2199, E-mail: walter.hubschmid@psi.ch and Schlamp et al. [6]. Walker et al. [4] used a single-mode probe laser to obtain flow velocities by observing the frequency shift. Maznev et al. described in [5] some specific arrangements for the heterodyne detection of LIGs. The heterodyne LIG technique was used by Schlamp et al. [8] to measure simultaneously sound speed and flow velocities. Electrostrictive LIGs for flow diagnostics were employed in [7, 9]. In [7] heterodyning was accomplished with diffraction at zero flow and in [9] with the superposition of two signal beams. Hart et al. [11] determined flow velocities from heterodynedetected electrostrictive LIGs, comparing experimental data with modelling in the frequency as well as in the time domain. Hemmerling et al. [13] applied the single-pulse heterodynedetected LIG technique to the investigation of the flow behind a rocket nozzle. Recirculation zones, predicted by numerical simulations, could be observed, using simplified signal analysis in the frequency domain (power spectrum). Advantages of analysing single-pulse LIG signals in the frequency domain and an example of gas-velocity measurements in pressurized reacting flows have been presented by Brown et al. [14]. They also discussed the effect of beam steering in inhomogeneous media.

We present in this paper the technique of electrostrictive LIGs for flow measurements, with heterodyne detection using a separate reference beam, and with data analysis in the frequency domain (power spectrum), as it had been applied in [13]. Properties and applicability of this technique, which allows for minimal and rapid signal post-processing, are described, and they are elucidated by simultaneous measurements of flow velocity and temperature in air jets behind a slot nozzle. For comparison, flow velocities are also determined with LDA, and with the LIG data when carrying out the evaluation in the time domain.

\section{Electrostrictive and thermal LIG technique with heterodyne detection}

Laser-induced gratings are spatially periodic transient modulations of the complex refractive index. They are usually generated as a response to the interference pattern formed by two short-pulse pump laser beams of the same wavelength $\lambda_{\mathrm{p}}$ and with wave vectors $\boldsymbol{k}_{1}$ and $\boldsymbol{k}_{2}$, which intersect at an acute angle $\theta$.

Electrostrictive laser-induced gratings can be generated at any frequency of the pump beams. The electric field polar- 
izes the dielectric medium, and the spatial inhomogeneity of the field of the interference pattern exerts the so-called electrostrictive force on the atoms or molecules. As a result, by pulsed excitation two sound-wave packets of equal amplitudes and with opposite signs are generated; see e.g. [15]. To avoid destructive superposition, the duration of the pulses should be short compared with the acoustic period. The wavelength $\Lambda$ is defined by the fringe spacing of the interference pattern, $\Lambda=\lambda_{\mathrm{p}} /(2 \sin (\theta / 2))$. The wave packets propagate in the directions of the grating vectors $\boldsymbol{q}$ and $-\boldsymbol{q}$, which are lying normal to the fringes of the electromagnetic field intensity generated by the pump beams. The equations $\pm \boldsymbol{q}= \pm\left(\boldsymbol{k}_{2}-\right.$ $\boldsymbol{k}_{1}$ ) hold, with $\boldsymbol{q}=2 \pi / \Lambda$. In case of excitation with a multimode laser, in order to obtain an efficient LIG, the difference in path lengths of the two laser beams has to be within their coherence length.

The sound-wave packets are detected by diffracting a cw (probe) laser beam with a wavelength $\lambda_{0}$ (frequency $\omega_{0}$ ), directed at the Bragg angle $\theta_{\mathrm{B}}=\arcsin \left(\lambda_{0} / 2 \Lambda\right)$ to the optical axis (bisectrix of the angle $\theta$ ). The coupling between electromagnetic and acoustic waves generates the frequencies $\omega_{1,2}=\omega_{0} \pm \Omega_{\mathrm{a}}$ in the diffracted beam [16]. Here $\Omega_{\mathrm{a}}$ denotes the frequency of the acoustic waves. For small dissipation, as is the case in our experiments, we may write $\Omega_{\mathrm{a}}=q v_{\mathrm{s}}$, where $v_{\mathrm{s}}$ is the adiabatic sound velocity; see the discussion in [17]. The total signal beam, at the same angle $\theta_{\mathrm{B}}$ to the optical axis as the incident probe beam, results from the superposition of the beams diffracted at the two travelling sound-wave packets. Dissipation of the sound waves is caused by viscosity and heat conduction (see e.g. [15]), with dissipation decay rates proportional to $\Lambda^{-2}$. Therefore, to reduce dissipation, the angle $\theta$ should be chosen small. Besides dissipation, the finite transverse dimensions of the probe volume also limit the duration of the LIG signal.

If the grating is formed in a flow with velocity $\boldsymbol{v}$, the two sound waves experience a Doppler shift and the frequencies of the diffracted beam become $\omega_{1,2}=\omega_{0} \pm q v_{\mathrm{s}}+\boldsymbol{q} \boldsymbol{v}$. However, also in this case, we have $\omega_{1}-\omega_{2}=2 \Omega_{\mathrm{a}}$, so that the measurement of the time-dependent intensity from Bragg scattering does not contain information on the flow velocity, as frequencies observable in such a scheme originate from interference of waves. This is explicitly shown in the derivation given below. To determine the flow velocity, a heterodyne-detection scheme is needed ${ }^{1}$. The reference beam may originate, as shown in [7], from diffraction of the probe beam in a region with vanishing flow. More flexible, however, is the use of an additional beam. Its frequency $\omega_{3}$ may be shifted by $\Delta \omega_{\mathrm{s}}=\omega_{0}-\omega_{3}$ with respect to the frequency of the probe beam, as e.g. done in [13]. The beam is adjusted to pass the LIG at the crossing point of the pump beams and to become superimposed to the diffracted probe beam. The advantage of shifting the frequency will become clear in the discussion of the power spectrum of the signal.

Thus, by applying the equation of acousto-optical coupling (the wave equation of an electric field with spatially dependent permittivity due to density waves) [16], one obtains for the field strength $E(t)$ of the signal beam reaching the de-

\footnotetext{
${ }^{1}$ Schlamp et al. [6] made use of beam misalignments to determine flow velocities with homodyne detection.
}

tector, with the field strength of the reference beam added:

$$
\begin{aligned}
E(t)= & A \exp \left[-t / \tau_{\mathrm{a}}-(t-\bar{\tau})^{2} / \tau^{\prime^{2}}\right] \\
& \times \sin \left[\left(\omega_{0}-\Omega_{\mathrm{a}}+\boldsymbol{q} \boldsymbol{v}\right) t+\alpha_{1}\right] \Theta(t) \\
& -A \exp \left[-t / \tau_{\mathrm{a}}-(t+\bar{\tau})^{2} / \tau^{\prime \prime 2}\right] \\
& \times \sin \left[\left(\omega_{0}+\Omega_{\mathrm{a}}+\boldsymbol{q} \boldsymbol{v}\right) t+\alpha_{1}\right] \Theta(t) \\
& +B \sin \left[\left(\omega_{0}-\Delta \omega_{\mathrm{s}}\right) t+\alpha_{2}\right]
\end{aligned}
$$

The amplitude $A$ characterizes the coupling constant of the scattering of the probe wave at the sound-wave packets (the change of sign in the first two terms is due to the opposite signs of the two acoustic waves), $B$ is the constant amplitude of the reference wave, $\alpha_{i}$ are phase angles, which have random values, and $t=0$ is the time of the grating formation, denoted by the Heaviside function $\Theta(t)$. The unit pulse function of the reference wave is not written explicitly, as it is broader than the decay function from the acoustic propagation and dissipation. Only the projection of $\boldsymbol{v}$ onto $\boldsymbol{q}, v_{q}$, enters (1); it is the component of the flow velocity that can be measured.

The temporal decay of the Bragg-diffracted waves is taken into account by introducing in (1) (a) $\tau_{\mathrm{a}}$ from acoustic dissipation and (b) the transit times $\tau^{\prime}$ and $\tau^{\prime \prime}$ of the acoustic waves out of the probe volume, which is assumed to have a Gaussian shape. $\tau^{\prime}$ and $\tau^{\prime \prime}$ are related by $\tau^{\prime \prime} / \tau^{\prime}=\left(v_{\mathrm{s}}+v_{q}\right) /\left(v_{\mathrm{s}}-v_{q}\right)$. The time interval $\bar{\tau}$, also introduced in (1), takes into account that, in general, it cannot be arranged that the probe beam meets symmetrically the two sound-wave packets. The acoustic decay time $\tau_{\mathrm{a}}$, being larger in the experiment (about $0.9 \mu \mathrm{s}$ ) than the acoustic transit time (about $0.35 \mu \mathrm{s}$ ), is no longer explicitly noted in the following. The radiation intensity $I(t)$ is defined, up to a constant factor, as the square of the electric field strength, averaged over optical periods. One obtains the various terms of $I(t)$ according to the familiar rule that a superposition of electric field strengths in the signal beam,

$C_{i} \sin \left[\left(\varpi+\Omega_{i}\right) t+\alpha_{i}\right]+C_{k} \sin \left[\left(\varpi+\Omega_{k}\right) t+\alpha_{k}\right]$,

generates from interference the time-dependent term

$C_{i} C_{k} \cos \left[\left(\Omega_{i}-\Omega_{k}\right) t+\Delta \alpha\right]$

in the expression for the radiation intensity $I(t)$. Thereby, $\varpi$ is considered to be an optical frequency, and $\Omega_{i}$ and $\Omega_{k}$ are low frequencies; i.e. there is no averaging over the respective periods. Furthermore, by definition $\Delta \alpha=\alpha_{i}-\alpha_{k}$.

Thus, one finds for the radiation intensity at the detector, neglecting the constant contribution from the reference beam alone, that

$$
I(t) \propto\left[\begin{array}{c}
\exp \left[-(t-\bar{\tau})^{2} / \tau^{\prime 2}\right] \cos \left(\Omega_{1} t+\Delta \alpha\right) \\
-\exp \left[-(t+\bar{\tau})^{2} / \tau^{\prime \prime 2}\right] \cos \left(\Omega_{2} t-\Delta \alpha\right) \\
+(A / 2 B)\left[\begin{array}{c}
\exp \left[-2(t-\bar{\tau})^{2} / \tau^{\prime 2}\right] \\
+\exp \left[-2(t+\bar{\tau})^{2} / \tau^{\prime \prime 2}\right] \\
-2 \exp \left[-(t-\bar{\tau})^{2} / \tau^{\prime 2}\right. \\
\left.-(t+\bar{\tau})^{2} / \tau^{\prime 2}\right] \cos \Omega_{0} t
\end{array}\right]
\end{array}\right] \Theta(t),
$$


where

$\Omega_{0}=2 \Omega_{\mathrm{a}}=2 q v_{\mathrm{s}}, \quad \Omega_{1} \equiv \Omega_{\mathrm{a}}-\Delta \omega_{\mathrm{s}}-\boldsymbol{q} \boldsymbol{v}$,

$\Omega_{2} \equiv \Omega_{\mathrm{a}}+\Delta \omega_{\mathrm{s}}+\boldsymbol{q} \boldsymbol{v}, \quad \Delta \alpha=\alpha_{2}-\alpha_{1}$.

Equation (3) confirms that the signal, without any reference beam added, oscillates with the frequency $\Omega_{0}$ only. The terms in (3), which carry the information on the flow velocity, may be enhanced with respect to the $\Omega_{0}$ term by an intensity of the reference beam large compared with the intensity of the diffracted beam.

In the case of $\bar{\tau}=0$, and $v_{q} \ll v_{\mathrm{s}}$, which means that the equation $\tau^{\prime 2}=\tau^{\prime \prime 2} \equiv \tau^{2}$ holds approximately, one obtains a somewhat simplified expression for $I(t)$. It shows more directly the modulation (with frequency $\Delta \omega_{\mathrm{s}}+q v_{q}$ ) and the effect of $\Delta \alpha$ :

$$
\begin{aligned}
I(t) \propto & \left\{\exp \left(-t^{2} / \tau^{2}\right) \sin \Omega_{\mathrm{a}} t \sin \left[\left(\Delta \omega_{\mathrm{s}}+q v_{q}\right) t-\Delta \alpha\right]\right. \\
& \left.+(A / B) \exp \left(-2 t^{2} / \tau^{2}\right) \sin ^{2} \Omega_{\mathrm{a}} t\right\} \Theta(t) .
\end{aligned}
$$

The frequencies $\Omega_{0}, \Omega_{1}$, and $\Omega_{2}$, which carry the physical information on the measured flow (4), can be determined for given data by fitting the experimental curve $I(t)$ to the model curve of (3).

In this paper, the procedure mainly described is to determine the frequencies $\Omega_{i}$ by spectral analysis. Both the cosine transform $I_{\mathrm{c}}(\Omega)$ and the sine transform $I_{\mathrm{S}}(\Omega)$ are, however, strongly $\Delta \alpha$-dependent, even for a single term (with one frequency). For the power spectrum $P(\Omega) \equiv I_{\mathrm{c}}^{2}(\Omega)+$ $I_{\mathrm{s}}^{2}(\Omega)$, which is used by us, the $\Delta \alpha$-dependence enters only if there is interference of different frequencies. The integrals needed in the expression for $P(\Omega)$ can be expressed by known functions [18]. Qualitatively, neglecting any interference with other peaks, the term with oscillation at frequency $\Omega_{1}$ has the form

$P(\Omega) \propto \tau^{\prime 2} \exp \left[-\frac{\left(\Omega-\Omega_{1}\right)^{2} \tau^{\prime 2}}{2}\right]+O\left(\Omega-\Omega_{1}\right)^{2}$.

More precisely, $P(\Omega)$ includes, besides the Gaussian function, as given above, other terms of power two and higher even powers. They cause a slower decay of the tails than from the Gaussian alone. Besides peaks at the positive frequencies $\Omega_{i}$, the power spectrum also has analogous terms at the negative frequencies $-\Omega_{i}$.

In the approximation that all interference terms depending on different frequencies $\Omega_{i}$ can be neglected, because of their frequency separation being large enough, the local maxima of the power spectrum $P(\Omega)$ for the function $I(t)$ are situated at the frequencies $\pm \Omega_{i}, i=0-2$, and at $\Omega=0$. Otherwise, the peak positions are slightly shifted from the frequencies $\Omega_{i}$, the shift being dependent on $\Delta \alpha$. In the case of the $\Omega_{1}$ and $\Omega_{2}$ oscillations, the interference is supposed to be small for $\left(\tau^{\prime} / \sqrt{2}\right)^{-1},\left(\tau^{\prime \prime} / \sqrt{2}\right)^{-1} \ll \Omega_{2}-\Omega_{1}$. The detailed analysis shows that $P(\Omega)$ contains contributions independent of $\Delta \alpha$, contributions proportional to $\cos (2 \Delta \alpha)$, and contributions proportional to $\sin (2 \Delta \alpha)$.

The quantities of interest can be obtained in a straightforward manner from the frequencies $\Omega_{i}$. First, from calibration measurements at zero flow, one obtains the frequency shift $\Delta \omega_{\mathrm{s}}$ and the grating vector $q$, using the relations $\Delta \omega_{\mathrm{s}}\left(v_{q}=0\right)=\left(\Omega_{2}-\Omega_{1}\right) / 2$ and $q=\Omega_{0}\left(T_{0}\right) / 2 v_{\mathrm{s}}\left(T_{0}\right)$, which follow from (4). In this way, usually, more accurate values for $\Delta \omega_{\mathrm{s}}$ and $q$ are obtained than from the setting of the frequency shifter $\left(\Delta \omega_{\mathrm{s}}\right)$ and from geometric measurements $(q)$. The value for the adiabatic sound velocity $v_{\mathrm{s}}\left(T_{0}\right)$ at the temperature $T_{0}$ is taken from the literature.

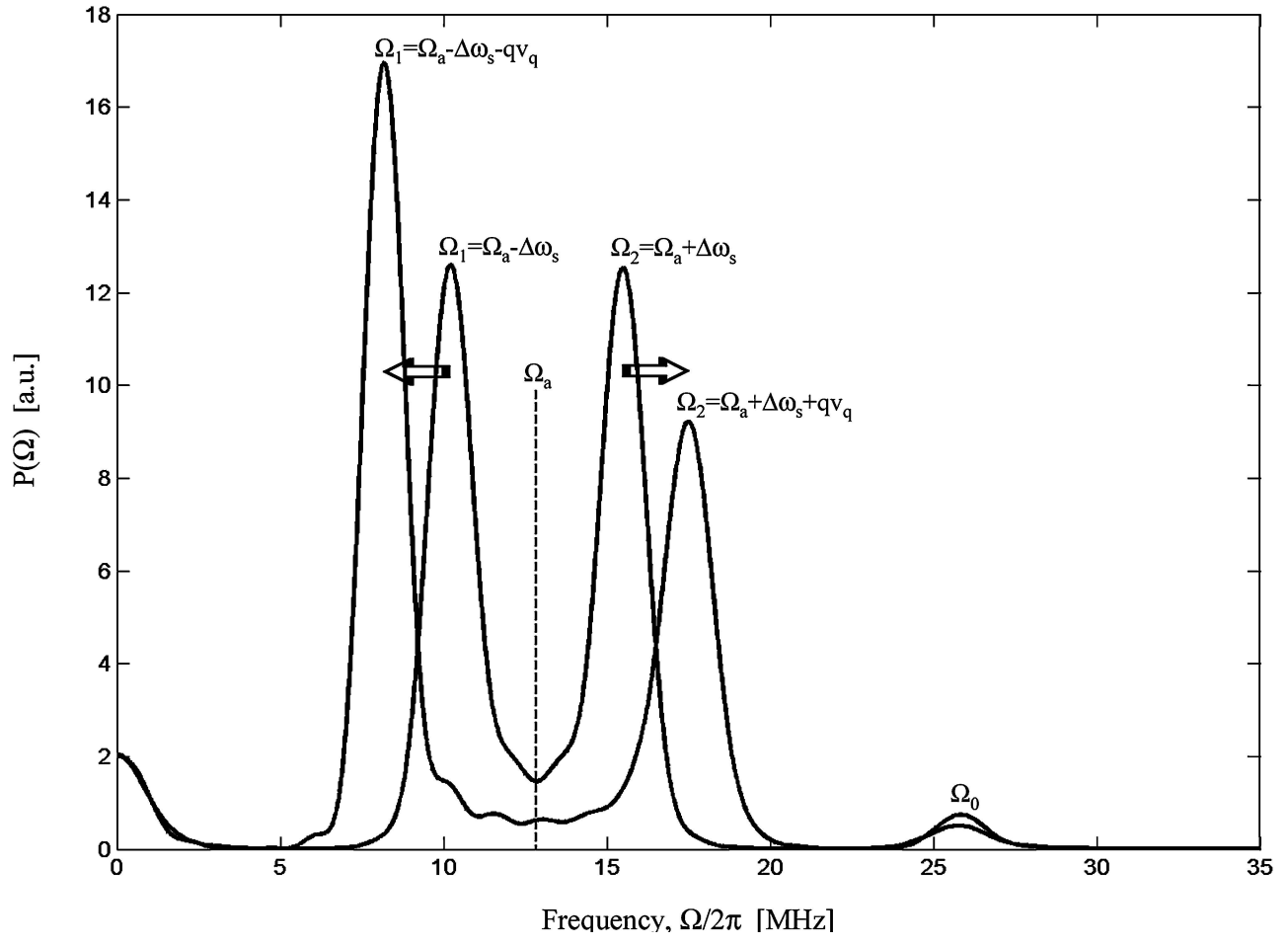

FIGURE 1 General form of the power spectrum of a heterodynedetected LIG signal from numerical modelling; a spectrum for quiescent gas at $T_{0}=295 \mathrm{~K}$ and its modification with flow $\left(v_{q}=40 \mathrm{~m} \mathrm{~s}^{-1}\right)$ are shown. The parameters are $\Omega_{\mathrm{a}}\left(T_{0}\right) / 2 \pi=13.4 \mathrm{MHz}, \Delta \omega_{\mathrm{s}} / 2 \pi=$ $2.2 \mathrm{MHz}, A / B=0.2, \Delta \alpha=0, \tau^{\prime}=$ $0.41 \mu \mathrm{s}$ (for $v_{q}=0$ ), $v_{\mathrm{s}}=345 \mathrm{~m} / \mathrm{s}$, and $\bar{\tau}=0$ 
The flow component $v_{q}$ in the direction of $\boldsymbol{q}$ is determined according to the relation

$v_{q}=\frac{\Omega_{2}-\Omega_{1}-2 \Delta \omega_{\mathrm{s}}}{2 q}$,

which follows from (4). Finally, the sound velocity $v_{\mathrm{s}}(T)$ at the location of the measurement is determined from $\Omega_{0}=$ $\Omega_{1}+\Omega_{2}$ :

$v_{\mathrm{s}}(T)=\frac{\Omega_{1}(T)+\Omega_{2}(T)}{2 q}$.

Thus, if gas composition and specific heat capacities do not change in the domain investigated, the local temperature is obtained from the ideal gas relation between sound velocity and temperature:

$T=\left(\frac{v_{\mathrm{s}}(T)}{v_{\mathrm{s}}\left(T_{0}\right)}\right)^{2} T_{0}=\left(\frac{\Omega_{0}(T)}{\Omega_{0}\left(T_{0}\right)}\right)^{2} T_{0}$

Figure 1 gives the general picture of how the power spectrum depends on the quantities $\Omega_{\mathrm{a}}, \Omega_{0}=2 \Omega_{\mathrm{a}}, \Delta \omega_{\mathrm{s}}$, and $v_{q}$. It should be emphasized that in the case of a non-zero shift $\Delta \Omega_{\mathrm{s}}$ between the frequencies of the probe and reference beams, the two peaks at $\Omega_{1}$ and $\Omega_{2}$ in the power spectrum of the photodetector signal exist even in quiescent gas. In a flow the peaks are shifted due to the Doppler effect, as related to their initial positions in the quiescent gas: with the increase of the flow velocity in the direction of $\boldsymbol{q}$ the peaks move apart while, if the flow has the opposite direction, the peaks move closer towards each other. Hence, both the value and the sign of the local flow-velocity component can be derived from the power spectrum in this case.

The application of thermal gratings for flow-velocity measurements can be discussed along similar lines. By the absorption of light, in addition to counterpropagating sound waves (Brillouin modes), the non-propagating Rayleigh mode is generated in the medium [19]. In the case that the absorbed radiation is instantaneously converted into heat, the $E$-field at the detector from diffraction at the Rayleigh mode has the form:

$E(t)=C \exp \left(-t / \tau_{\mathrm{b}}\right) \cos \left[\left(\Omega_{0}+\boldsymbol{q} \boldsymbol{v}\right) t+\alpha_{1}\right] \Theta(t)$,

where $\tau_{\mathrm{b}}$ is the dissipative decay time of the Rayleigh mode. The case of non-instantaneous heat deposition is treated in [20].

\section{$3 \quad$ Experimental set-up \\ 3.1 Set-up for heterodyne-detected LIG}

A pulsed Nd:YAG laser (Continuum NY-81, $\lambda_{\mathrm{p}}=$ $1064 \mathrm{~nm}$, pulse energy $\approx 50 \mathrm{~mJ}$, repetition rate $20 \mathrm{~Hz}$, pulse
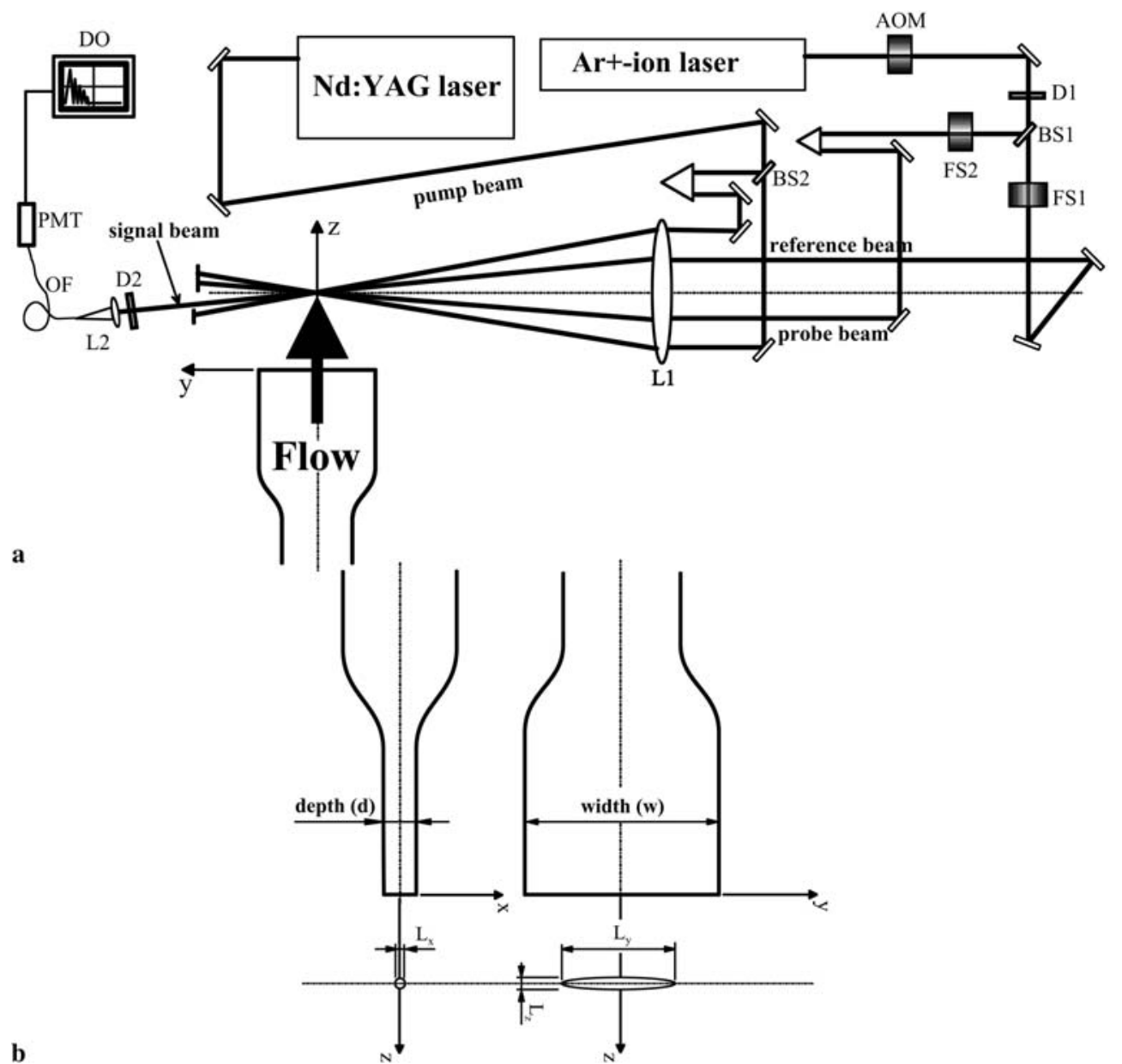

FIGURE 2 (a) Scheme of the experimental set-up. BS: beam splitters; FS1,2: acousto-optical frequency shifters; LS1,2: focusing lenses; D1,2: iris diaphragms; FB: optical fibre; PMT: photomultiplier tube; DO: digital oscilloscope; AOM: acousto-optical modulator of the probe and the reference beams. (b) Relative arrangement of a nozzle and the probe volume in the laboratory-fixed Cartesian coordinate system 
duration $\approx 8 \mathrm{~ns}$, coherence length $\approx 8 \mathrm{~mm}$ ) provided the two equally polarized pump beams crossing at $\theta=2.26^{\circ}$ to form an electrostrictive grating with $\Lambda \approx 27.0 \mu \mathrm{m}$ in a standard experimental arrangement, as shown in Fig. 2a. $\Lambda$ and $\theta$ are calculated from the grating constant $q=2.32 \times 10^{5} \mathrm{~m}^{-1}$, which was determined in a zero-flow measurement (cf. Sect. 4.1). The corresponding acoustic decay time [15] is about $0.9 \mu \mathrm{s}$. The excitation beams, as well as the probe and reference beams, were focused by the same lens $(f=750 \mathrm{~mm})$. The diameter of the focal region of the pump beams was about $0.25 \mathrm{~mm}$. Diameters of probe and reference beams in the focal region of the pump beams were slightly larger.

The beam of an $\mathrm{Ar}^{+}$laser (Coherent Innova 90, $\lambda_{0}=$ $514 \mathrm{~nm}$, power $\approx 0.9 \mathrm{~W}$ ) probed the laser-induced grating. The probe beam satisfied the Bragg condition in twodimensional forward phase-matching geometry. The reference beam was adjusted in the plane of the excitation and the probe beams, symmetrically to the probe beam with respect to the optical axis, to overlap the beam diffracted by the LIG. Two dc-voltage-controlled acousto-optical frequency shifters (Brimrose, TEF-80-30-514) introduce a frequency difference of $\Delta \omega_{\mathrm{s}} / 2 \pi \approx 2.75 \mathrm{MHz}$ between the probe and the reference beams. This frequency shift was determined by a LIG measurement at zero flow (see Sect. 4.1). The overlapping diffracted and reference beams were focused into a multimode optical fiber (core diameter $120 \mu \mathrm{m}$ ), which was employed as a spatial filter to reduce background light of incoherently scattered probe radiation. Flow velocities that can be measured with the set-up are between about $-30 \mathrm{~m} \mathrm{~s}^{-1}$ (this value depends on the chosen frequency shift $\Delta \omega_{\mathrm{s}}$ ) and about $+200 \mathrm{~m} \mathrm{~s}^{-1}$.

The temporally resolved heterodyne signals were detected by a photomultiplier (PMT, Philips XP2020). To avoid saturation of the PMT by the high cw light level of the reference beam, an acousto-optical modulator (AOM, model ISOMET M1205C-2) was employed to gate the $\mathrm{Ar}^{+}$laser, synchronously with the excitation laser pulses, during the period of about $2 \mu \mathrm{s}$. The gate width was selected to be larger than the acoustic transit times $\tau^{\prime}, \tau^{\prime \prime}$ (about $0.35 \mu \mathrm{s}$ in our experimental configuration, see below). The gate time defines the time $t=-t_{0}(t=0$ is defined by the time of the grating formation, at the centre of the Nd:YAG pulse). The signal beam is recorded using a digital oscilloscope (Tektronix, TDS $744 \mathrm{~A}$ ) with a full bandwidth of $500 \mathrm{MHz}$ and a sampling rate of $1 \mathrm{GHz}$.

\section{2}

\section{Model air jets}

Two different slot nozzles were used in our experiments to generate jets of air at atmospheric pressure, the first nozzle (A) was used for the temperature measurements, the second (B) for comparative measurements of the LIG with the LDA technique. Both nozzles had an aperture formed by compressing the end of a copper tube. The aperture of nozzle A had a cross section at the exit of $S_{\mathrm{N}} \approx 32.5 \mathrm{~mm}^{2}$ (width $w=22 \mathrm{~mm}$ and depth $d=1.5 \mathrm{~mm}$ ). The deformed part of the nozzle was $20 \mathrm{~mm}$ long. A resistance heater wrapped around the tube provided temperatures up to $1100 \mathrm{~K}$. Wall and gas temperatures were measured by thermocouples. Fine grids of copper wires were placed inside the nozzle to enable homoge- neous heating of the flow, and this prevented its use to seed the flow with particles. Non-heatable nozzle B, of similar length to A, had slightly smaller orifice dimensions. The cross section at the exit was $S_{\mathrm{N}} \approx 29 \mathrm{~mm}^{2}$ (width $w=18 \mathrm{~mm}$ and depth $d=1.7 \mathrm{~mm}$ ).

The flow rate was measured with a flow meter (Vögtlin V300). In our experiments the flow varied between $19.51 / \mathrm{min}$ and $69 \mathrm{l} / \mathrm{min}$ at room temperature. This corresponded to average flow velocities between $11.2 \mathrm{~m} \mathrm{~s}^{-1}$ and $40 \mathrm{~m} \mathrm{~s}^{-1}$. Taking the depth $d$ as the characteristic length, the range of Reynolds numbers is calculated to have values between about $1.2 \times 10^{3}$ and $4.3 \times 10^{3}$.

The axes of our laboratory-fixed Cartesian coordinate system were defined in the following way (Fig. 2b): the $z$ axis was coincident with the symmetry axis of the nozzle and was pointing in the main flow direction, while the $x$ and $y$ axes were lying in the plane of the nozzle orifice, the $y$ axis being parallel to its wide side. Hence, the origin of the coordinate system was at the centre of the orifice. The pump and probe beams were adjusted to lie in the $y-z$ plane, with their crossing point (centre of the focal volume) located on the $z$ axis, in such a way that the optical axis was parallel to the $y$ axis and the grating vector $\boldsymbol{q}$ was coincident with the $z$ axis.

The measurements in the flow were performed with the probe volume centred at the axis of the jet, at various distances from the plane of the nozzle orifice. We define the transverse extension $L_{x}=L_{z}$ of the probe volume to correspond to the waist diameter of the pump beams and obtain $L_{x}=L_{z} \approx 0.25 \mathrm{~mm}$ for our experimental set-up. As the crossing angle between the pump beams is small $\left(\theta \approx 2.26^{\circ}\right)$, a large extension of the probe volume in the $y$ direction $\left(\equiv L_{y}\right)$ is obtained even in the case of focused beams. We estimate $L_{y}$ to be about $10 \mathrm{~mm}$. The extensions $L_{x}$ and $L_{y}$ of the probe volume are therefore small compared with the depths and widths, respectively, of the nozzles used in the experiment, and inhomogeneity of the flow velocity in the probe volume is small for laminar flows.

\section{$4 \quad$ Results and discussion \\ 4.1 Procedure of data analysis}

For each measurement a series of 100 single-shot LIG signals containing 1000 points with a time step of $1 \mathrm{~ns}$ (i.e. the duration of one signal is $1 \mu \mathrm{s}$ ) has been recorded. The heterodyne time-resolved LIG signal is Fourier transformed, in order to obtain the power spectrum. Two peaks at (approximately) $\Omega_{1}$ and $\Omega_{2}$ dominate the power spectrum; the third peak (at frequency $\Omega_{0}$ ) is clearly smaller, because $A \ll B$ (cf. (3)). An automatic peak-search algorithm can be applied to the power spectrum to derive the peak positions and to calculate the flow velocity $v_{q}$ using (6). The mean velocity and its standard deviation can be obtained from the velocity histogram, which is approximated by a Gaussian distribution.

The time-resolved single-pulse heterodyne LIG signals (cf. Fig. 3) are sitting on the top of a rectangular pulse (not shown in the figure), which is introduced by gating the reference beam with an AOM as described in Sect. 3.1. The detected signals are strings of $10^{3}$ values, taken in steps of $1 \mathrm{~ns}$, i.e. they have a total duration of $1 \mu$ s. Their power spectrum $P(\Omega)$ is represented by the dots (marked by squares in 


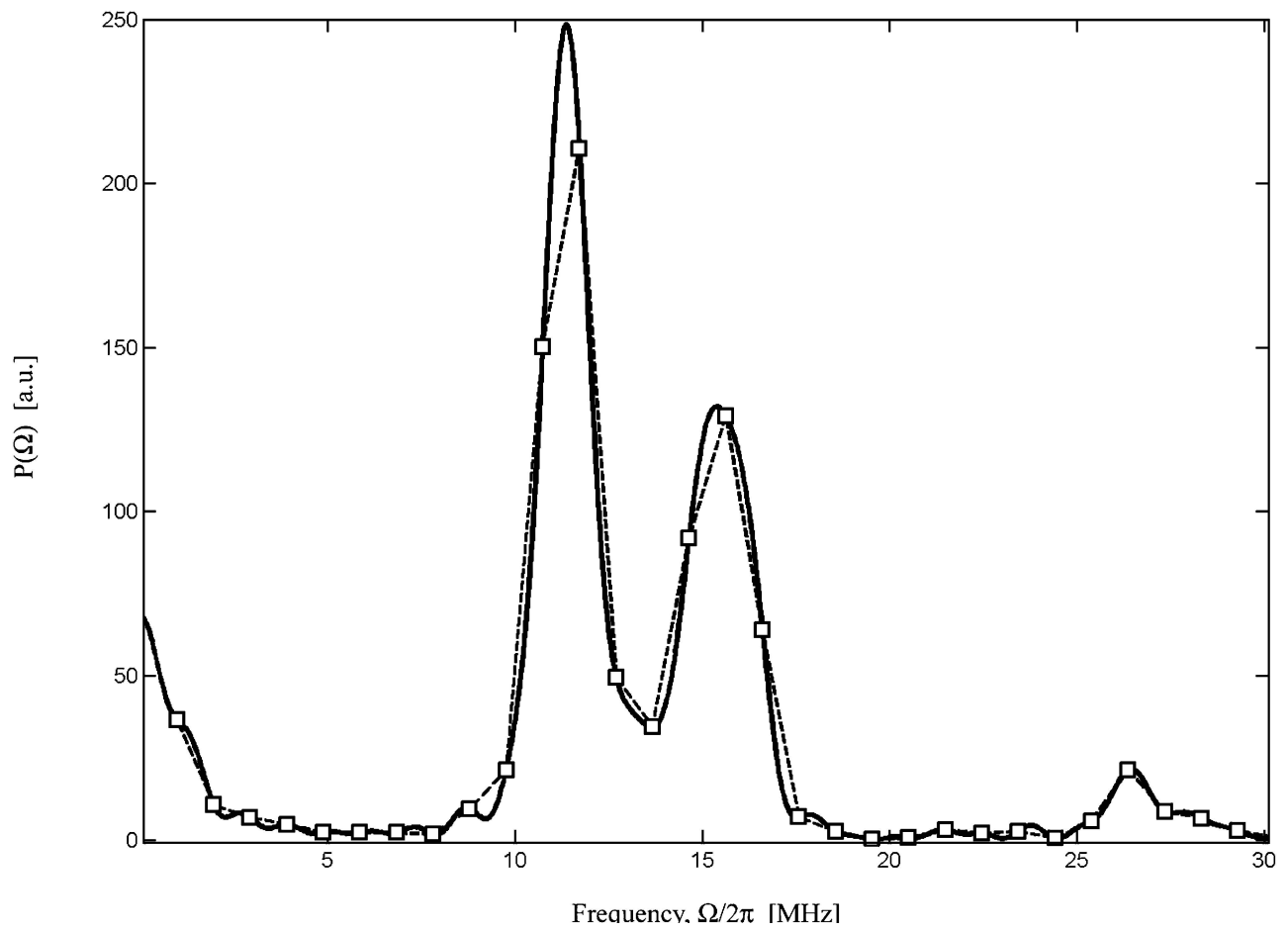

FIGURE 3 Influence of zero filling on power spectrum. Dashed line: without zero filling. Solid line: with zero filling

Fig. 3), which are connected in the figure by a dashed line. In order to obtain a denser set of points in the power spectrum, a data string of $2^{15}$ values is generated, where the values added are zeros (zero filling, cf. [21]). This improves the accuracy of determining positions of peaks in the spectrum, as a smoother curve of the power spectrum (solid line in Fig. 3) is obtained. With the number of $2^{15}$ sampling points a spacing of $0.03 \mathrm{MHz}$ from point to point was provided. To get a final resolution of $0.003 \mathrm{MHz}$, additionally a spline interpolation was applied.

Note that by truncating the recorded signal before it has fully decayed, the addition of zeros to the original data generates a discontinuity in the curve. As the Fourier transform of a step function is a sinc function, additional peaks, so-called sinc wiggles, appear in the Fourier spectrum. However, usu- ally, except in the case of experiments with weak signals, the amplitudes of these wiggles are small compared to those of the main peaks.

The power spectrum at zero flow provides approximate data for the experimental parameters $q$ (and therewith $\left.\Omega_{\mathrm{a}}\right), \Delta \omega_{\mathrm{s}}, \bar{\tau}, A / B, \tau^{\prime}$, and $\tau^{\prime \prime}$. They were used as initial values to fit the time-dependent curves $I(t)$ (at zero flow) to the model functions (3). The optimization, applying the Levenberg-Marquard scheme, with holding fixed all parameters but one in the iterations, gave the following values: $q=2.32 \times 10^{5} \mathrm{~m}^{-1}, \Delta \omega_{\mathrm{s}} / 2 \pi=2.75 \times 10^{6} \mathrm{~s}^{-1}, \bar{\tau}=41 \mathrm{~ns}$, $A / B=0.25$, and $\tau^{\prime}=\tau^{\prime \prime}=0.347 \mu \mathrm{s}$. The transit times $\tau^{\prime}$, $\tau^{\prime \prime}$ correspond to a propagation path of the sound waves of $0.12 \mathrm{~mm}$, and the time $\bar{\tau}$ to a misalignment of $14 \mu \mathrm{m}$ with respect to the symmetry axis of the probe volume. The ratio
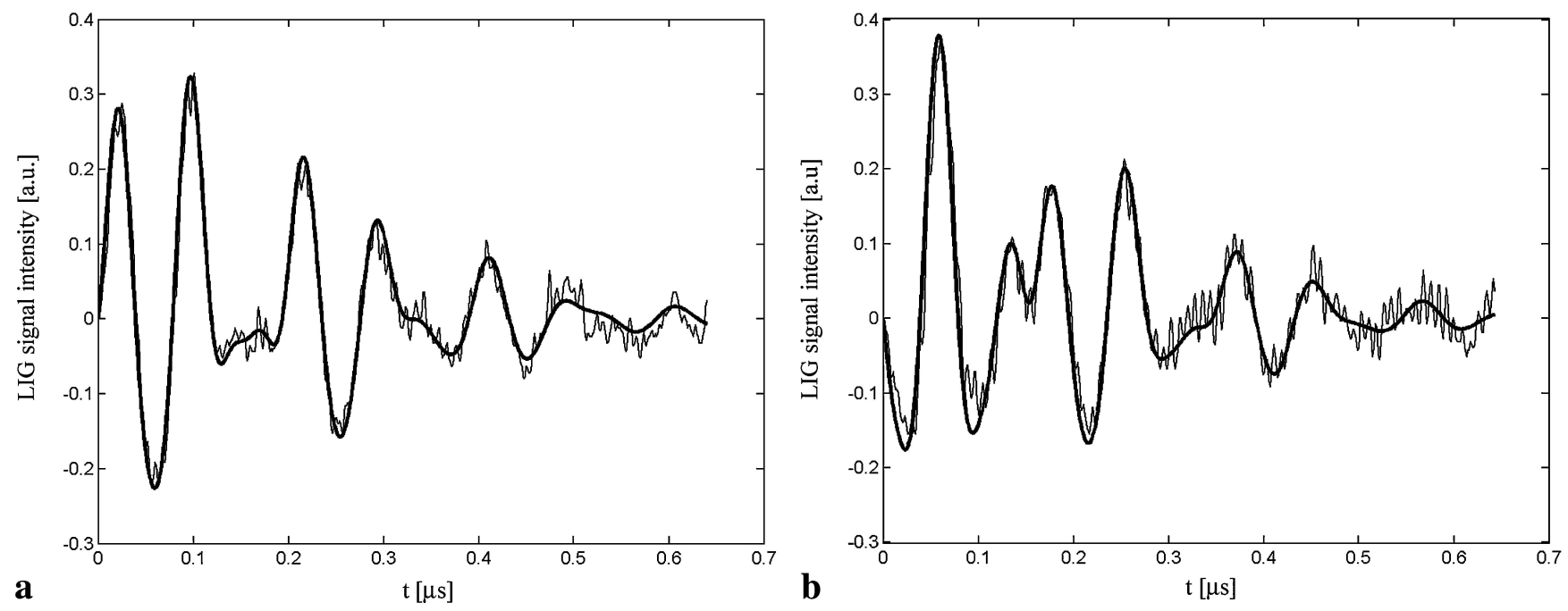

FIGURE 4 Two examples of experimental curves $I(t)$ for $v_{q}=0$ and their fits with model curves. The data are (a) $\Delta \alpha=-0.35$ and (b) $\Delta \alpha=2.58$ 
$\left(\tau^{\prime} / \sqrt{2}\right)^{-1} /\left(\Omega_{2}-\Omega_{1}\right)$, defined in Sect. 2 , is approximately equal to 0.11 . However, as peaks in the power spectrum have slower decay in the wings than Gaussian peaks, it may be supposed that the flow velocities $v_{q}$ obtained from the peak positions in the power spectrum still depend in an observable amount on the random value of $\Delta \alpha$. Actually, numerical analysis shows that the variations of $\Delta \alpha$ generate movements of the peaks in the power spectrum, which correspond to $v_{q}$ fluctuations of about $\pm 5 \mathrm{~m} \mathrm{~s}^{-1}$. The difference for the mean value of $\Delta \omega_{\mathrm{s}}$, obtained from power-spectrum analysis (average over $\Delta \alpha)$ and from fits with $I(t)$, is small, however. It corresponds to a deviation in $v_{q}$ of $0.4 \mathrm{~m} \mathrm{~s}^{-1}$. From fitting $I(t)$ the triggering time of the photomultiplier, $-t_{0}$, is also determined. Figure $4 \mathrm{a}$ and $\mathrm{b}$ show that the experimental curves $I(t)$ fit very well with the model functions if the optimized values of $\Delta \alpha$ are inserted.

\section{Flow velocities from LIG and LDA}

We determined by LIG, as described above, the flow velocities at various positions in the flow field of nozzle B. The measurement points were on the nozzle symmetry axis at distances of $z=1.17 d, z=2.35 d$, and $z=4.7 d$ from the nozzle exit. To check the accuracy of the results from the LIG technique, we compared the results with results obtained from LDA. These latter measurements were performed at the Swiss Federal Institute of Technology in the Laboratory for Thermodynamics. A standard LDA set-up was used. The measurement volume was a sphere with a diameter of about $80 \mu \mathrm{m}$. The mean diameter of the $\mathrm{TiO}_{2}$ seeding particles was $1 \mu \mathrm{m}$. To assure that repeated measurements were at the same positions inside the jet, a special mask was used for adjusting the location of the probe volume.

The mean values of the flow velocity, obtained by LDA measurements and by LIG measurements - from quick fits with the power spectrum and, for the distance $z / d=1.17$, also from fits with the model signal intensity $I(t)$ - agree well for lower velocities and small distances from the nozzle (Table 1). Results from LDA are, however, systematically larger when the turbulence in the flow is supposed to become relevant (at larger distances from the outlet and higher velocities). Data for the fits with the model signal intensity $I(t)$ are obtained by selecting at random 10 measurements for each flow condition (except for zero flow, and for the flow $11.2 \mathrm{~m} \mathrm{~s}^{-1}$ at $z / d=1.17: 20$ measurements). Standard deviations are clearly smaller for the LDA measurements than for LIG. LIG standard deviations are about constant for flows supposed to be dominantly laminar. A considerable part of the standard deviations for the power spectrum (quick fits with the peak positions) is due to the dependence on the random parameter $\Delta \alpha$, which is not accounted for in this technique. Doing fits in the time domain and taking into account the influence of the relative phase angle $\Delta \alpha$ achieve a clear improvement. The standard deviations are about one-half $\left(1.6-2.6 \mathrm{~m} \mathrm{~s}^{-1}\right)$ those for quick fits with the power spectrum. The improved singlepulse accuracy is of the same order as was earlier achieved with a set-up using two counterpropagating signal beams and data analysis in the time domain [9]. With the onset of turbulence standard deviations for LIG measurements as well as for LDA increase. The comparison of the results for $z / d=1.17$ between LIG (quick fits from the power spectrum) and LDA is illustrated in Fig. 5.

\subsection{Simultaneous temperature and flow-velocity measurements in heated jets}

We report in this subsection the measurement of temperatures, simultaneous with the flow velocity, which were obtained from the power spectrum of single-pulse signal intensities of heterodyne LIG. The sum of the frequencies $\Omega_{1}$ and $\Omega_{2}$, determined by a peak-search algorithm (Sect. 4.1), equals $\Omega_{0}$, which is according to (7) and (8) related to the sound velocity, and therefore to the temperature. The LIG temperature measurements were performed at $z / d=1.17$ of the jets from nozzle A, the air being heated to between $295 \mathrm{~K}$ and $525 \mathrm{~K}$. The values of the air temperatures were obtained by a thermocouple placed at the flow axis near the exit of the nozzle. During the measurements the flow rate was kept constant at $19.5 \mathrm{l} / \mathrm{min}$ of the unheated air. Temperatures determined by LIG agree within 1-2\% with the temperatures from the thermocouple, as one recognizes from the results presented in Fig. 6a. The relative standard deviations for $T$ are of the order of $1 \%$ in the lower-temperature domain $(300-400 \mathrm{~K})$. The deviations are clearly smaller than for each of the frequencies $\Omega_{1}$ and $\Omega_{2}$ separately. For $525 \mathrm{~K}$ they increase to $5 \%$. Fluctuations of $\Omega_{0}\left(\equiv \sigma_{\Omega_{0}}\right)$ result from the dependence of the power spectrum on random pulse-topulse variations of the phase shift $\Delta \alpha\left(\sigma_{\alpha}\right)$ and from LIG signal noise $\left(\sigma_{\mathrm{n}}\right)$. Detailed calculations show that the contribution of random variations of $\Delta \alpha$ to $\sigma_{\Omega_{0}}$ is much smaller than for $\sigma_{\Omega_{2}}-\Omega_{1}$ so that, presumably, $\sigma_{\mathrm{n}}$ contributes the dominating part to $\sigma_{\Omega_{0}}$. Difficulties in determining peaks with the search algorithm occurred at the highest temperature. This contributed in addition to the noted standard deviation for the temperature.

The expected linear growth of flow velocities with temperature can be observed from the results of simultaneous measurements of gas temperature and flow velocity (Fig. 6b). With the enhancement of temperature the gas density of the

\begin{tabular}{|c|c|c|c|c|c|c|}
\hline & $\begin{array}{l}\text { Mean outlet } \\
\text { flow velocity }\end{array}$ & 11.2 & 17 & 23.5 & 32 & 40 \\
\hline \multirow[t]{3}{*}{$z / d=1.17$} & $\mathrm{LIG}_{\mathrm{ps}}$ & $16.8 \pm 3.8$ & $25.3 \pm 3.5$ & $32.2 \pm 4.4$ & $43.2 \pm 3.2$ & $53.6 \pm 3.8$ \\
\hline & $\mathrm{LIG}_{\text {fit }}$ & $16.4 \pm 2.1$ & $25.7 \pm 1.6$ & $34.3 \pm 2.1$ & $44.4 \pm 2.6$ & $56.7 \pm 2.1$ \\
\hline & LDA & $16.3 \pm 0.3$ & $24.7 \pm 0.5$ & $34.4 \pm 0.6$ & $45.9 \pm 0.5$ & $58.4 \pm 0.6$ \\
\hline \multirow{2}{*}{$z / d=2.35$} & $\mathrm{LIG}_{\mathrm{ps}}$ & $16.0 \pm 3.2$ & $23.7 \pm 4.0$ & $32.2 \pm 4.5$ & $39.9 \pm 5.2$ & $50.8 \pm 7.6$ \\
\hline & LDA & $16.2 \pm 0.3$ & $24.7 \pm 0.4$ & $34.4 \pm 0.6$ & $45.8 \pm 0.7$ & $58.3 \pm 0.9$ \\
\hline \multirow[t]{2}{*}{$z / d=4.7$} & $\mathrm{LIG}_{\mathrm{ps}}$ & $12.5 \pm 5.6$ & $18.5 \pm 3.7$ & $27.7 \pm 6.4$ & $34.0 \pm 6.4$ & $42.0 \pm 6.3$ \\
\hline & LDA & $15.7 \pm 0.6$ & $23.8 \pm 2.1$ & $33.3 \pm 3.9$ & $43.7 \pm 5.4$ & $55.0 \pm 6.2$ \\
\hline
\end{tabular}

TABLE 1 Air-flow velocities in centre of air jet measured by LIG and LDA at distances $z / d$ from outlet of the air jet. All flow velocities are in $\mathrm{m} \mathrm{s}^{-1}$. $\mathrm{LIG}_{\mathrm{ps}}$ : quick fit from power spectrum of LIG signal. $\mathrm{LIG}_{\mathrm{fit}}$ : fit with time-dependent LIG signal intensity 


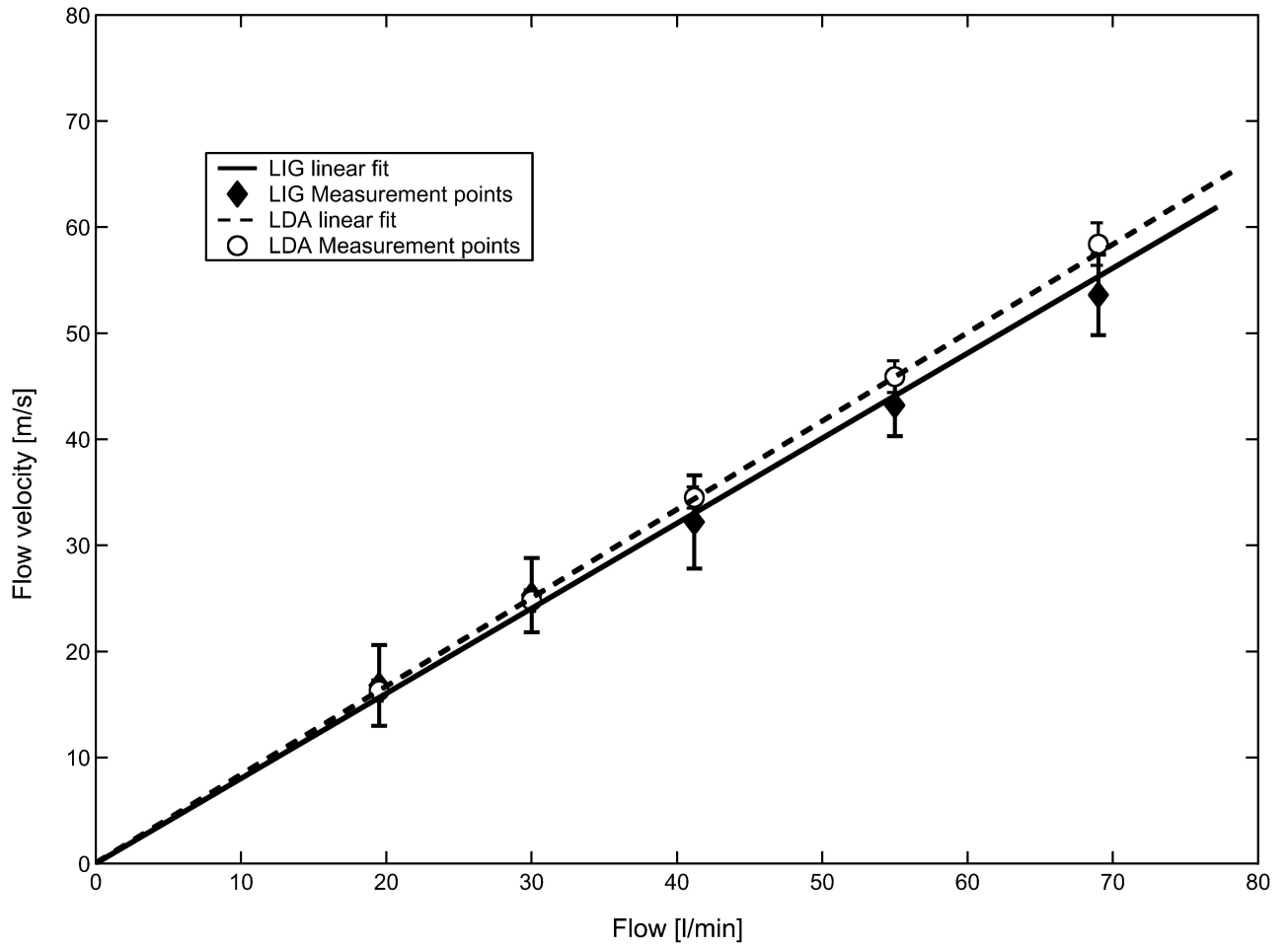

FIGURE 5 Flow-velocity measurements in air jets from LIG (quick fits with power spectrum) and from LDA, with standard deviations $(z / d=1.17)$

medium at constant pressure decreases and, as a consequence, the diffracted beam intensity decreases quadratically with density. In addition, lower gas density and higher temperature result in smaller values of the acoustic transit times $\tau^{\prime}, \tau^{\prime \prime}$ and of the damping time $\tau_{\mathrm{a}}$, and hence in a smaller number of visible peaks in the temporal evolution of the LIG signal. The two factors provide an increase of uncertainty in the determination of the oscillation period and larger standard deviations of measured flow velocities. To extend the upper limit of measurable temperatures, changes in the set-up should be provided. Especially, the diameter of the probe beam in the region of the acoustic waves should be enlarged to obtain longer observation times.

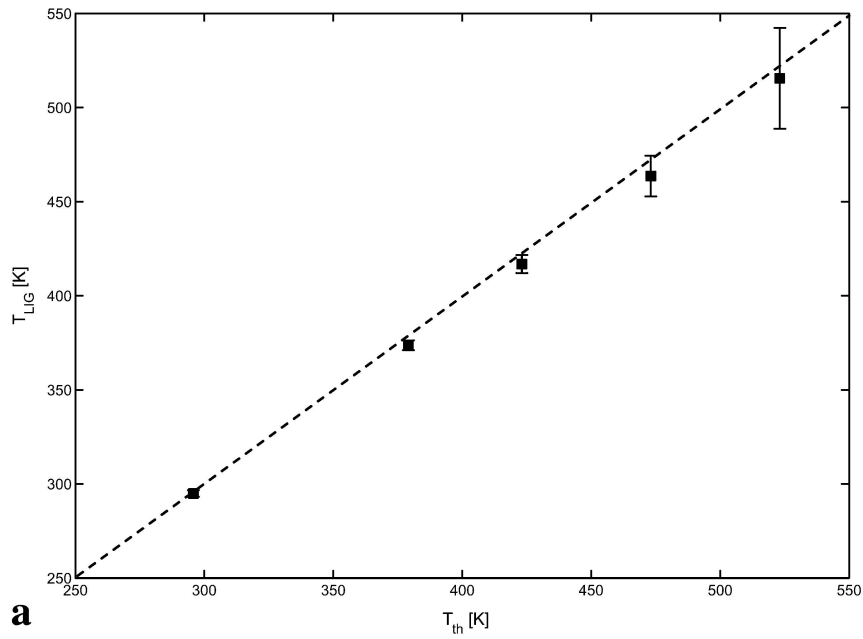

5

Conclusion

We discussed in this paper conditions of using the electrostrictive laser-induced grating technique with heterodyne detection as a non-intrusive technique, with moderate post-processing, for simultaneous single-shot measurements of flow velocity and temperature. The heterodyne LIG technique, as implemented by us, employs (1) a reference beam, which has its frequency shifted in relation to the probe beam, and (2) data analysis with quick fits from peaks of the power spectrum of the LIG signal intensity. Laboratory measurements of flow velocity and temperature in free-air jets behind a slot nozzle were performed, with flow velocities in the range

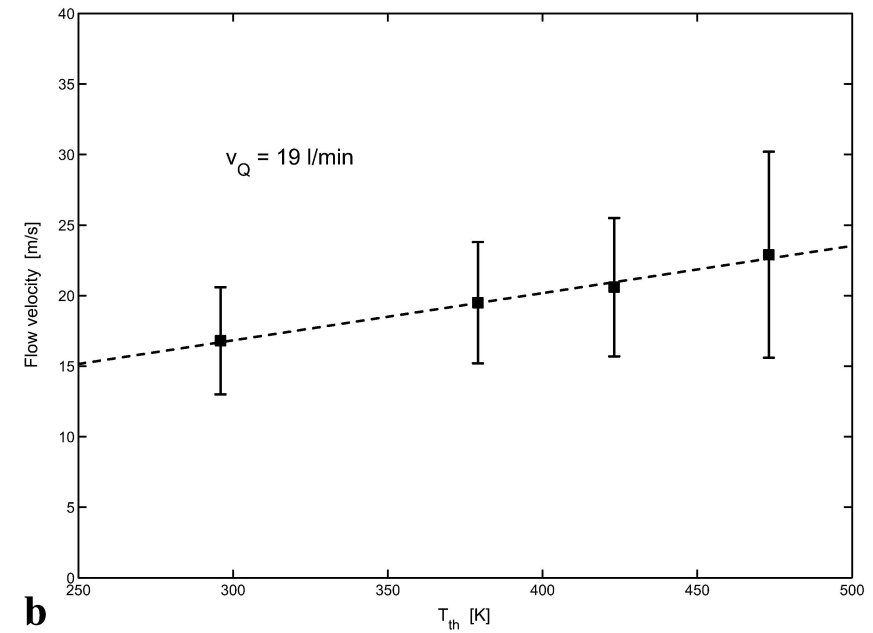

FIGURE 6 Simultaneous temperature and flow-velocity measurements using LIG versus temperatures measured by thermocouple $T_{\text {th }}$ (for constant flow rate of $19.5 \mathrm{l} / \mathrm{min}$ and at $z / d=1.17$ ). (a) Temperature $T_{\mathrm{LIG}}$; the dashed line represents the line of equal temperatures. (b) Flow velocity; the dashed line shows the linear fit 
of $15-60 \mathrm{~m} \mathrm{~s}^{-1}$ and temperatures up to $525 \mathrm{~K}$, to test the applicability and measurement accuracy of the technique. The main findings are:

1. Using quick fits from the power spectrum of the singlepulse LIG signal, standard deviations of the flow velocities near the nozzle exit are of the order of $4 \mathrm{~m} \mathrm{~s}^{-1}$. The values of flow velocities obtained from the power-spectrum analysis were compared with the results obtained by fitting the temporal shape of the same single-pulse LIG signals to model functions. Mean values given by the two approaches are similar within the standard deviations. The direct fitting of the signal intensity gives smaller standard deviations (about $1.5-2.5 \mathrm{~m} \mathrm{~s}^{-1}$ ) than the quick fits from the power spectrum. A considerable part of the standard deviations in the latter case is due to the dependence on the random phase between the reference beam and the Bragg-diffracted wave, which is not accounted for in the quick fits. The shorter the observation time (given by the acoustic transit and/or dissipation time), the more strongly this unconsidered random phase affects the measurement accuracy.

2. The flow velocities provided by the heterodyne LIG technique were compared with the results of LDA measurements using the same flows. Mean values of the derived flow velocities near the nozzle orifice are close for both techniques, while the standard deviations of LDA-defined values are significantly smaller than those of LIG measurements. Under conditions where turbulence is assumed to be stronger (larger distances from the nozzle orifice and higher velocities), a larger discrepancy between the LIGand LDA-defined mean values and an increase of standard deviations for both LIG and LDA measurements are observed.

3. For temperature measurements using the heterodynedetected LIG technique (with power-spectrum analysis), the mean values agree within $1-2 \%$ with values provided by a thermocouple inserted into the flow. The relative standard deviations, at the lower temperatures, are of the order of $1 \%$.
ACKNOWLEDGEMENTS The authors are grateful to A. Wokaun for the continuous support of the work. D.N. Kozlov, from the General Physics Institute in Moscow, is acknowledged for his help in performing the LIG measurements, and L. Blum, from the Laboratory of Thermodynamics of the Swiss Federal Institute of Technology (ETHZ), for his help with the LDA measurements. We thank T. Dreier for careful reading of the manuscript. Financial support by the Swiss Federal Office of Energy (BFE) and the ERCOFTAC Leonhard Euler Centre, Switzerland, is gratefully acknowledged.

\section{REFERENCES}

1 A. Eder, B. Dürst, M. Jordan: 'Laser Doppler Velocimetry'. In: Optical Measurements, 2nd edn, ed. by F. Mayinger, O. Feldmann (Springer, Berlin 2001)

2 W. Merzkirch: 'Particle Image Velocimetry'. In: Optical Measurements, 2nd edn, ed. by F. Mayinger, O. Feldmann (Springer, Berlin 2001)

3 E.B. Cummings, I.A. Leyva, H.G. Hornung: Appl. Opt. 34, 3290 (1995)

4 D.J.W. Walker, R.B. Williams, P. Ewart: Opt. Lett. 23, 1316 (1998)

5 A.A. Maznev, K.A. Nelson, J.A. Rogers: Opt. Lett. 23, 1319 (1998)

6 S. Schlamp, E.B. Cummings, H.G. Hornung: Appl. Opt. 38, 5724 (1999)

7 D.N. Kozlov, B. Hemmerling, A. Stampanoni-Panariello: Appl. Phys. B 71, 585 (2000)

8 S. Schlamp, E.B. Cummings, T.H. Sobota: Opt. Lett. 25, 224 (2000)

9 B. Hemmerling, D.N. Kozlov, A. Stampanoni-Panariello: Opt. Lett. 25, 1340 (2000)

10 S. Schlamp, E. Allen-Bradley: 'Homodyne detection laser-induced thermal acoustics velocimetry', AIAA Paper (American Institute of Aeronautics and Astronautics) 2000-0376, Reno, Nevada, USA (2000)

11 R.C. Hart, R.J. Balla, G.C. Herring: Appl. Opt. 40, 965 (2001)

12 S. Schlamp, T. Rösgen: 'Experimental considerations for laser-induced thermal acoustics (LITA) in compressible turbulent flows', AIAA Paper 2003-3454, $33^{\text {rd }}$ AIAA Fluid Dynamics Conference, Orlando, FL, USA, June 23-26 (2003)

13 B. Hemmerling, M. Neracher, D.N. Kozlov, W. Kwan, R. Stark, D. Klimenko, W. Clauss, M. Oschwald: J. Raman Spectrosc. 33, 912 (2002)

14 M.S. Brown, Y. Li, W.L. Roberts, J.R. Gord: Appl. Opt. 42, 566 (2003)

15 A. Stampanoni-Panariello, B. Hemmerling, W. Hubschmid: Phys. Rev. A 51, 655 (1995)

16 R.B. Boyd: Nonlinear Optics (Academic, San Diego 1992)

17 W. Hubschmid, R. Bombach, B. Hemmerling, A. StampanoniPanariello: Appl. Phys. B 62, 103 (1996)

18 I.S. Gradshteyn, I.M. Ryzhik: Table of Integrals, Series and Products (Academic, San Diego 1980) Formula 3.896

19 W. Hubschmid, B. Hemmerling, A. Stampanoni-Panariello: J. Opt. Soc. Am. B 12, 1850 (1995)

20 W. Hubschmid, B. Hemmerling: Chem. Phys. 258, 109 (2000)

21 W.H Press, B.P. Flannery, S.A. Teukolsky, W.T. Vetterling: Numerical Recipes (Cambridge University Press 1988) 